\title{
Should we genotype the sperm of fathers from patients with 'de novo' mutations?
}

\author{
Eric Pasmant ${ }^{1,2}$ and Laurence Pacot ${ }^{1,2}$ \\ 'Service de Génétique et Biologie Moléculaires, Hôpital Cochin, HUPC, Assistance Publique-Hôpitaux de Paris, Paris, \\ France and 'Institut Cochin, INSERM U1016, Université Paris Descartes, CARPEM, Paris, France
}

Correspondence should be addressed to E Pasmant Email

eric.pasmant@ parisdescartes.fr

\begin{abstract}
In this issue of the European Journal of Endocrinology, Lin et al. report a de novo heterozygous nonsense variant in the PHEX gene in an X-linked hypophosphatemic rickets patient. The authors described a germline mosaicism in the sperm of the unaffected father of the proband, providing the opportunity to discuss the concept of isolated germline mosaicism. In addition to the genetic information passed on from generation to generation, each of us is born with a small number of novel genetic changes -de novo mutations- that occurred either prezygotically or postzygotically. When de novo mutational events occur prezygotically, mutation may pre-exist in a parent who is mosaic but the mutation might be inherited in the zygote and potentially in all cells of the developing offspring resulting in a de novo disease phenotype. Only somatic cells (mainly from blood and skin) are routinely used in genetic analyses, and a hint about possible germline mosaicism can be obtained only if somatic mosaicism is detected, or if more affected siblings are born with an identical de novo variant. There is now a wide range of disorders for which the occurrence of parental germline mosaicism has been reported. Interestingly, Lin et al. analyzed eight different tissues of the proband's father (including sperm), which allowed a conclusive assessment of his mosaicism extension. This study suggests that sperm analysis could more often be performed in our routine genetic screening for germline mosaicism in fathers of patients with apparently de novo mutations.
\end{abstract}

European Journal of

Endocrinology

(2019) 182, C1-C3

In this issue of the European Journal of Endocrinology, Lin et al. report a de novo heterozygous nonsense variant in the PHEX gene in a X-linked hypophosphatemic rickets patient (1). Lin et al. described a germline mosaicism in the sperm of the unaffected father of the proband, providing the opportunity to discuss the concept of isolated germline mosaicism.

Upon fertilization, a human zygote inherits half of its genome from the mother via the oocyte and the other half from the father through the sperm. In addition to the genetic information passed on from generation to generation, each of us is born with a small number of novel genetic changes - de novo mutations - that occurred either prezygotically or postzygotically $(2,3,4)$. A typical human genome varies at 4-5 million positions compared with the human reference genome. All of this genetic variation must have occurred as a de novo germline mutation in an individual at least once in human evolution. The occurrence of novel mutations in each generation explains why reproductively lethal disorders continue to occur.

Recent genome and exome sequencing studies of parent-offspring trios have provided new insights into the number and distribution of de novo mutations in health and disease $(5,6)$. Historically, the germline mutation rate in humans has been calculated by analyzing the incidence of genetic disorders. Nowadays, next-generation sequencing (NGS) approaches in parent-offspring trios 
can be used to directly study the occurrence of all types of de novo mutations throughout the genome.

When de novo mutational events occur prezygotically, mutation may pre-exist in a parent who is mosaic (and usually unaffected), but the mutation might be inherited in the zygote and potentially in all cells of the developing offspring (constitutional i.e., non-mosaic) resulting in a de novo disease phenotype. There are different types of mosaicisms that describe which parts of the body harbor the mutated cells and the potential for transmission to offspring. These include isolated germline mosaicism (also called gonadal mosaicism), somatic mosaicism, and a combination of germline and somatic mosaicism (2, 3, 4). Although these classifications are useful in a practical sense, they cannot be conclusively assigned owing to the limitations of tissue sampling. Only somatic cells (mainly from blood and skin) are routinely used in genetic analyses, and a hint about possible germline mosaicism can be obtained only if somatic mosaicism is detected, or if more affected siblings are born with an identical de novo variant. Interestingly, Lin et al. analyzed eight different tissues of the proband's father (including sperm) by Sanger sequencing, which allowed a conclusive assessment of his mosaicism extension.

Because de novo prezygotic mutations arise in the parental germline before fertilization, their occurrence is intimately linked to the biology of germ cells. Moreover, the susceptibility to de novo mutations may be influenced by genetic factors such as nucleotide content, replication timing, distance to recombination hotspots, nucleosome occupancy, transcription, and chromatin openness. Several mechanisms at the origin of DNA mutation are known, most predominantly involving DNA replication which explains the increased ratio of de novo mutations on the paternal allele to the maternal allele, as there are many more germline cell divisions in spermatogenesis than in oogenesis. Spermatogonial stem cells divide through mitosis approximately every 16 days, maintaining the spermatogonial stem cell pool. By contrast, each menstrual cycle, a few oocytes complete the first meiotic division. Approximately $80 \%$ of all de novo germline point mutations arise on the paternal allele, and advanced paternal age at conception has been established as the major factor linked to the increase in the number of de novo mutations in the offspring. Furthermore, the study of large cohorts of parent-offspring trios provided insight into the distribution of mutations throughout the genome, the genomic context in which they arise, and possible underlying mechanisms $(6,7)$. These studies showed that the difference in biology of male and female gametogenesis gives rise to distinct mutational signatures in offspring that diverge with increasing parental age. Studies of de novo mutations in humans have estimated the mutation rate of single-nucleotide variants to be $\sim 10^{-8}$ mutations per generation, giving rise to $45-60$ de novo mutations per genome.

The developmental timing and cell lineage affected, combined with the phenotypic consequences of the mutation, ultimately determine the tissue distribution of mosaicism and also the patterns of disease reoccurrence within families. The risk for genetic defects increases linearly for some conditions, and exponentially for others. A striking increase with paternal age has been observed for a small subset of de novo mutations that are highly recurrent and localize to specific nucleotides in the genome. These de novo mutations are thought to grant spermatogonial stem cells a growth advantage, leading to clonal expansion of mutated cells in the testis. For instance, gain-of-function mutations in some genes in the RAS-MAPK pathway have been shown to cause clonal expansion of mutant spermatogonial stem cells owing to proliferative selective advantage. The first mutations implicated in clonal expansion in spermatogonial stem cells were initially shown to involve genes that cause developmental disorders such as Noonan and Costello syndrome (caused by PTPN11 and HRAS mutations, respectively), Apert, Crouzon, and Pfeiffer syndromes (FGFR2), achondroplasia, Muenke syndrome and thanatophoric dysplasia (FGFR3), and multiple endocrine neoplasia 2A and 2B (RET). Mutations that are positively selected at the spermatogonial stem cell level but are detrimental at the organism level are referred to as 'selfish mutations' (8). These pathogenic mutations, which cause developmental disorders that show an extreme paternal bias in origin and an epidemiological paternal age effect are identical to oncogenic driver mutations in tumors. In a process akin to oncogenesis, selfish gain-of-function mutations provide a selective advantage that may involve increasing the rate of symmetrical divisions of the mutant spermatogonia leading to their clonal expansion over time, which results in increased apparent mutation levels in sperm with age.

The identification of a de novo mutation as the cause of disease in a patient has several implications for the patient and his family. The detection of the genetic defect underlying the phenotype establishes a genetic diagnosis that can be used to provide a prognosis based on data from other patients with similar mutations and information about current treatment options. In terms of family planning, the identification of a de novo mutation 
as the cause of disease in a child can be positive news with regard to recurrence risk. Parents of an individual with a postzygotic mutation have a low risk of recurrence of the mutation in an additional child, estimated as being the same as the population risk.

The component of genetic counselling that addresses recurrence risks is challenging for patients with mosaic disorders both because it requires analysis of sample cells within a given tissue and because mosaicism may be tissue-specific or tissue-limited. As noted earlier, in the case of a couple who has an offspring with an apparently constitutional, de novo occurrence of a disorder that can also be inherited in an autosomal dominant pattern, one of the parents must be considered at risk for having germline mosaicism. Therefore, the couple is at risk of passing on the same mutation to additional offspring. Diagnosis of tissue-limited mosaicism is clearly challenging when the affected tissue is not blood or skin, which are the two tissues that are most frequently analyzed in clinical laboratories. In some cases, a vigilant clinician will request analysis of multiple tissues to rule out low-level mosaicism: blood, skin, saliva and any particular affected tissues being the most common. Detection relies on using sensitive genotyping techniques that can detect low-level mosaicism in a more routine fashion. Sensitive detection and quantification of mutations using powerful genomic tools like NGS or targeted strategies (e.g. droplet digital PCR) will soon become standard tools for the accurate estimation of recurrence risks in families for which a genetic disease is caused by an apparently de novo disease gene mutation event. However, the level of this risk is difficult to estimate for most disorders as it depends on the proportion of the germ cell progenitors that harbor the mutation. There is now a wide range of disorders for which the occurrence of parental germline mosaicism has been reported. The study by Lin et al. is an illustration of this condition. Moreover, this study also suggests that sperm analysis could more often be performed in our routine genetic screening for germline mosaicism in fathers of patients with apparently de novo mutations.

\section{Declaration of interest}

The authors declare that there is no conflict of interest that could be perceived as prejudicing the impartiality of this commentary.

\section{Funding}

This research did not receive any specific grant from any funding agency in the public, commercial or not-for-profit sector.

\section{Author contribution statement}

E P and L P drafted, edited, and approved the manuscript.

\section{References}

1 Lin Y, Cai Y, Xu J, Zeng C, Sheng H, Yu Y, Li X \& Liu L. Isolated germline mosaicism in the phenotypically normal father of a girl with X-linked hypophosphatemic rickets. European Journal of Endocrinology 2020182 K1-K4. (https://doi.org/10.1530/EJE-19-0472)

2 Biesecker LG \& Spinner NB. A genomic view of mosaicism and human disease. Nature Reviews: Genetics 201314 307-320. (https:// doi.org/10.1038/nrg3424)

3 Samuels ME \& Friedman JM. Genetic mosaics and the germ line lineage. Genes 20156 216-237. (https://doi.org/10.3390/ genes6020216)

4 Veltman JA \& Brunner HG. De novo mutations in human genetic disease. Nature Reviews: Genetics 201213 565-575. (https://doi. org/10.1038/nrg3241)

5 Acuna-Hidalgo R, Veltman JA \& Hoischen A. New insights into the generation and role of de novo mutations in health and disease. Genome Biology 201617 241. (https://doi.org/10.1186/s13059-0161110-1)

6 Goriely A. Decoding germline de novo point mutations. Nature Genetics 201648 823-824. (https://doi.org/10.1038/ng.3629)

7 Goldmann JM, Wong WS, Pinelli M, Farrah T, Bodian D, Stittrich AB, Glusman G, Vissers LE, Hoischen A, Roach JC et al. Parent-of-originspecific signatures of de novo mutations. Nature Genetics 201648 935-939. (https://doi.org/10.1038/ng.3597)

8 Goriely A \& Wilkie AO. Paternal age effect mutations and selfish spermatogonial selection: causes and consequences for human disease. American Journal of Human Genetics 201290 175-200. (https://doi.org/10.1016/j.ajhg.2011.12.017) 